\title{
Optimal Curvature and Gradient-constrained Directional Cost Paths in 3-Space
}

\author{
Alan J. Chang* Marcus Brazil ${ }^{\dagger} \quad$ J. Hyam Rubinstein ${ }^{\ddagger}$ \\ Doreen A. Thomas $\S$
}

\begin{abstract}
In the design of underground tunnel layout, the development cost is often dependent on the direction of the tunnel at each point due to directional ground fracturing. This paper considers the problem of finding a minimum cost curvature-constrained path between two directed points in 3-space, where the cost at every point along the path depends on the instantaneous direction. This anisotropic behaviour of the cost models the development cost of a tunnel in ground with faulting planes that are almost vertical. The main result we prove in this paper is that there exists an optimal path of the form $\mathcal{C S C S C S C}$ (or a degeneracy), where $\mathcal{C}$ represents a segment of a helix with unit radius and $\mathcal{S}$ represents a straight line segment. This generalises a previous result that in the restriction of the problem to the horizontal plane there always exists a path of the form $\mathcal{C S C S C}$ or a degeneracy which is optimal. We also prove some key structural results which are necessary for creating an algorithm which can construct an optimal path between a given pair of directed points in 3-space with a prescribed directional cost function.
\end{abstract}

Keywords computational geometry; path optimization; curvature-constrained paths; anisotropic costs.

${ }^{*}$ A. J. Chang, Department of Mechanical Engineering, The University of Melbourne, Victoria 3010, Australia, email: alancj.unimelb@gmail.com

${ }^{\dagger}$ M. Brazil (corresponding author), Department of Electrical and Electronic Engineering, The University of Melbourne, Victoria 3010, Australia, email: brazil@unimelb.edu.au

$\ddagger$ J. H. Rubinstein, Department of Mathematics and Statistics, The University of Melbourne, Victoria 3010, Australia, email: rubin@ms.unimelb.edu.au

$\S$ D. A. Thomas, Department of Mechanical Engineering, The University of Melbourne, Victoria 3010, Australia, email: doreen.thomas@unimelb.edu.au 


\section{Introduction}

The problem we address in this paper is that of finding a minimum cost path between two points in $\mathbb{R}^{3}$ where the cost at every point along the path depends on the instantaneous direction and the path satisfies the following constraints:

- the magnitude of the gradient at every point along the path does not exceed a given value $m$;

- the horizontal projection of the path satisfies a curvature constraint and has initial and final directions corresponding to prescribed initial conditions.

This 3-dimensional path optimisation problem models the basic requirements of the design of underground declines in hard rock mines accessing gold, copper, zinc, nickel or polymetallic deposits. In such mines, access from the surface to a collection of discrete ore bodies is provided by a complex infrastructure network of shafts and tunnels. In this paper, we focus on the geometry of individual tunnels, known as the declines, which are used to transport personnel and equipment and to haul ore and waste, usually by truck, to the surface. For vehicle navigability, the declines must satisfy a maximum curvature constraint (in the horizontal planar projection), typically to accommodate a turning circle radius of about $25 \mathrm{~m}$; and a gradient constraint, typically of at most 1:7 [4]. Furthermore, the construction cost of the decline depends on the direction of development, due to the anisotropic behaviour of the region. This behaviour is caused by directed faulting or fracturing of the ground which increases the tunneling and support costs depending on the extent to which the tunnel is aligned with the direction of fracturing [12] [11]. Furthermore, this anisotropic behaviour of the ground can be assumed to only depend on the direction of the tunnel in the horizontal projection, since the gradient of the tunnels is relatively flat. This assumption allows us to simplify the problem as follows.

Consider the problem of finding a minimum cost curvature-constrained path between two directed points in $\mathbb{R}^{2}$ where the cost at every point along the path depends on the instantaneous direction and the path must satisfy a minimum length constraint. In short, we wish to solve the following problem:

$$
\min _{E \in \mathbb{P}_{p q}} \int_{E} c(\alpha) \mathrm{d} s \text { subject to } \int_{E} \mathrm{~d} s \geq L_{l b}
$$

for some given directional cost function $c(\alpha)$ and $L_{l b} \geq 0$, where $\mathbb{P}_{p q}$ denotes the set of all curvature-constrained paths between two directed points $p$ and $q$ in the plane.

This planar path problem is able to solve the 3-dimensional path optimisation problem we are interested in. Given a maximum gradient $m$ and a vertical distance of 
$z$ between the two given points, we can reduce the 3 -dimensional problem into (1.1) with a lower bound on the length of the path of $L_{l b}=z / m$. The simpler case where development cost is isotropic was studied in [3].

In [6], it has been shown that there exists an optimal path of the form $\mathcal{C S C S C}$ (or degeneracy) if we do not consider the lower bound on length, where $\mathcal{C}$ denotes a continuous subset of the unit circle, and $\mathcal{S}$ represents a straight line segment. This result generalises Dubins original result for shortest curvature-constrained paths in [9], which is essentially the special case where $c(\alpha)=1$. In this paper, we build upon the techniques introduced in [6] to show that there exists an optimal path solving (1.1) of the form $\mathcal{C S C S C S C}$ (or degeneracy) with loops (if and when needed), where a loop is defined to be a unit circle, tangent to a point on the path, added to ensure the path satisfies the lower bound constraint. Note that loops do not correspond to a $\mathcal{C}$ label and are treated as additional components since they can be placed anywhere along the path. Given a CS-path $E$ and lower bound on length $L_{l b}$, it is clear that the correct number of additional loops to consider is the lowest number of loops required to make the CS-path with loops satisfy the minimum length constraint.

The results proven in this paper are useful for developing an algorithm which efficiently constructs an optimal network of underground mine declines in 3-dimensions. The most important geometric constraints of the decline are the curvature and gradient constraints. We model the anisotropic behaviour of rock within each geological domain with an anisotropic cost function. The theory developed in this paper enables us to incorporate anisotropic cost behaviour into the optimisation framework described in [3] while still accounting for the gradient and curvature constraints. This provides a more realistic cost model as it incorporates the ground conditions.

In Section 2, we will present some background from [6]. In Section 3.1, we extend the techniques introduced in [6] to be applicable to the problem studied in this paper. We then apply these techniques in Section 3.2 to show that there exists an optimal CS-path to the problem (1.1). In Section 3.3, we prove the main result of this paper, that there exists an optimal path of the form $\mathcal{C S C S C S C}$ (or degeneracy) with loops which solve (1.1). A result relating to the construction of optimal paths is shown in Section 3.4 to illustrate the difficulty of explicitly constructing an optimal path for a given velocity function, minimum length $L_{l b}$, and directed points $p, q$. Finally, we provide some examples of optimal paths in Section 4. 


\section{Background}

In this section, for completeness, we include the necessary background from [8] and $[6]$.

- $a, b \in \mathbb{R}^{2}$ and $p, q \in \mathbb{R}^{2} \times \mathbb{R} / 2 \pi \mathbb{Z}$ denote directed points.

- A $C^{k}$ function is a function whose first $k$ derivatives exist and are continuous.

- A path from $a$ to $b$ is a directed piecewise $C^{1}$ curve from $a$ to $b$. We parametrise our paths by arc-length, $E:\left[0, t_{f}\right] \rightarrow \mathbb{R}^{2}$ with $E(0)=a$ and $E\left(t_{f}\right)=b$.

- The direction $\alpha \in \mathbb{R} / 2 \pi \mathbb{Z}$ at a differentiable point of a path $E(t)$ is given by the polar angle of the tangent at the point in the direction of increasing $t$. At the endpoints, the directions are taken as the respective limits of $\alpha$.

- Given a minimum turning circle radius $R>0$, a curvature-constrained path between two directed points $p, q$ is a $C^{1}$ path that is piecewise $C^{2}$, where the absolute curvature everywhere along the path is bounded above by $1 / R$, and the given directions at the start and end points coincide with the directions of $p$ and $q$ respectively. Without loss of generality, we assume the minimum turning circle radius $R=1$ throughout this paper. Let $\mathcal{C}$ be a label denoting a continuous subset of the unit circle. Let $\mathcal{S}$ be a label denoting a straight line segment.

- A CS-path is a curvature-constrained path $E:\left[0, t_{f}\right] \rightarrow \mathbb{R}^{2}$ such that there exist $t_{0}, \cdots, t_{n}$ where $t_{0}=0$ and $t_{n}=t_{f}, t_{i-1}<t_{i}$ for $i=1, \cdots, n$, where $E$ is not twice differentiable at $t_{i}$ for $i=1, \cdots, n$, and each subpath $E_{i}:\left[t_{i-1}, t_{i}\right] \rightarrow$ $\mathbb{R}^{2}$ is either a $\mathcal{C}$ arc or $\mathcal{S}$ segment. The form of such a CS-path is then the sequence of $\mathcal{C}$ and $\mathcal{S}$ labels in ascending order of $i$. The sense of a $\mathcal{C}$ can be further specified using the labels $\mathcal{L}$ and $\mathcal{R}$ for left-turning and right-turning arcs respectively. In the form of a CS-path, any consecutive $\mathcal{C}$ arcs must be of opposite sense due to the condition that the CS-path is differentiable but not twice differentiable at the point in between two consecutive labels. This condition also implies that there will never be consecutive $\mathcal{S}$ labels in the form of a CS-path.

Dubins [9] first studied the problem of finding a shortest curvature-constrained path between any two given directed points in $\mathbb{R}^{2}$ and proved the following theorem.

Theorem 2.1 Given any two directed points $p, q \in \mathbb{R}^{2} \times \mathbb{R} / 2 \pi \mathbb{Z}$, a shortest curvatureconstrained path is of the form $\mathcal{C S C}, \mathcal{C} \overline{\mathcal{C}}$ or a degeneracy where $\overline{\mathcal{C}}$ denotes a $\mathcal{C}$ arc of length greater than $\pi$. 


\subsection{Directional Cost}

Let $\mathbb{R}_{+}$denote the set of all strictly positive real numbers.

A directional cost function is a continuous, piecewise $C^{2}$ function $c: \mathbb{R} / 2 \pi \mathbb{Z} \rightarrow$ $\mathbb{R}_{+}$. The corresponding velocity function $v: \mathbb{R} / 2 \pi \mathbb{Z} \rightarrow \mathbb{R}_{+}$is given by $v(\alpha)=$ $1 / c(\alpha)$, which is also continuous and piecewise $C^{2}$. The interpretation of the velocity function in the context of directional cost is a measure of the distance that can be travelled in the direction $\alpha$ at the cost of one unit. In this paper, to simplify a proof of a key result, we will add an additional condition that the size of the derivative of the directional cost function (where differentiable) is bounded.

Given a path $E$ and a directional cost function $c$, we have the following definitions:

- the length of $E, L(E)$, is given by $L(E)=\int_{E} \mathrm{~d} s$;

- $E$ is degenerate if it has zero length;

- the cost of $E, T(E)$, is given by $T(E)=\int_{E} c(\alpha) \mathrm{d} s$.

Let $\mathbb{P}_{p q}$ denote the set of all curvature-constrained paths between two directed points $p$ and $q$. An optimal path from $p$ to $q$ is a path $E \in\left\{P \in \mathbb{P}_{p q}: T(P) \leq T(Q)\right.$ $\left.\forall Q \in \mathbb{P}_{p q}\right\}$.

The following theorem, which is a generalisation of Theorem 2.1, was proven in [6] for (1.1) without the lower bound on length.

Theorem 2.2 Given any two directed points $p, q \in \mathbb{R}^{2} \times \mathbb{R} / 2 \pi \mathbb{Z}$ and velocity function $v$, there exists an optimal path from $p$ to $q$ of the form $\mathcal{C S C S C}$, or a degeneracy of $\mathcal{C S C S C}$.

\section{$2.2 \quad$ Reversible directions}

This paper makes use of CS-path deformations and reversible directions introduced in [6]. We state the critical definitions and results here.

The degree $d$ of a CS-path is the number of straight line segments and inflection points between two consecutive arcs in the path. Each of these represents a distinct interval or point at which a reversible CS-path deformation can be performed. A CS-path is reducible if it can be deformed to a CS-path of strictly lower degree with cost no greater than the original CS-path.

Let $E$ be a CS-path from $p$ to $q$ as shown in Figure 1(a). Let $r$ be a point along an $\mathcal{S}$ subpath of $E$ in the direction $\alpha_{r}$. We can form a new CS-path $E^{\prime}$ by fixing 
the path from $p$ to $r$, extending the $\mathcal{S}$ subpath in direction $\alpha_{r}$ by length $\delta$, and then translating the remainder of the original path (from $r$ to $q$ ) by $\delta$ in the direction $\alpha_{r}$ so that the new path ends up at $q^{\prime}$ as shown in Figure 1(a). The important property of this deformation is that it is reversible, i.e. we can contract the $\mathcal{S}$ subpath by $\delta$ instead, resulting in a path ending at $q^{\prime \prime}$.

We can perform similar reversible deformations at inflection points in $\mathcal{C C}$ subpaths. Let $r$ be an inflection point in an $\mathcal{R} \mathcal{L}$ subpath as shown in Figure $1(\mathrm{~b})$. We can form a new CS-path by fixing the path from $p$ to $r$, then introducing an $\mathcal{L}$ arc of length $2 \gamma$ starting with direction $\alpha_{r}$, followed by an $\mathcal{R}$ arc of length $2 \gamma$ starting with direction $\alpha_{r}+2 \gamma$. The new path ends up at $q^{\prime}$, a directed point with the same direction as $q$, but translated $4 \sin \gamma$ away from $q$, in the direction $\alpha_{r}+\gamma$. We can perform a contraction by shortening the existing $\mathcal{L} \mathcal{R}$ subpath starting from $r$, until both arcs are $2 \gamma$ shorter. The extension and contraction at an inflection is defined this way so that $q^{\prime}$ and $q^{\prime \prime}$ are antipodal with respect to $q$ as seen in Figure 1(b). By considering the mirror images of these figures, we get the corresponding actions for the situation where, instead, $r$ is an inflection point in an $\mathcal{L} \mathcal{R}$ subpath.

Since the effect of a reversible deformation is determined by whether it occurs on a $\mathcal{S}$ subpath, $\mathcal{L} \mathcal{R}$ inflection point or $\mathcal{R} \mathcal{L}$ inflection point, it is convenient to define the following term:

Definition 2.3 Given a CS-path with degree $d$, let $\alpha_{i}$ for $i=1, \cdots$, d denote the directions of the straights and inflection points in the order traversed by the path. We define $A_{i}=\left(\alpha_{i}, \rho_{i}\right)$ for $i=1, \cdots, d$ to be reversible directions, where

$$
\rho_{i}=\left\{\begin{array}{cl}
-1 & \text { if it corresponds to the inflection point in an } \mathcal{L} \mathcal{R} \text { subpath } \\
0 & \text { if it corresponds to a straight } \mathcal{S} \text { subpath; } \\
1 & \text { if it corresponds to the inflection point in an } \mathcal{R} \mathcal{L} \text { subpath }
\end{array}\right.
$$

A pair of reversible directions $A_{i}, A_{j}(i \neq j)$ are repeated if $A_{i}=A_{j}$ (i.e. $\alpha_{i}=\alpha_{j}$ and $\rho_{i}=\rho_{j}$ ). A set of reversible directions are said to be distinct if there are no repeated reversible directions in the set. In Figure $1, \delta$ and $\gamma$ were used to represent positive quantities for illustrative purposes. Since the deformations are reversible, it is natural to let $\delta_{i}<0$ and $\gamma_{i}<0$ represent the contractions of length $\left|\delta_{i}\right|$ and $\left|\gamma_{i}\right|$ while $\delta_{i}>0$ and $\gamma_{i}>0$ represent extensions. For brevity, we adopt the following notation:

- $\delta_{i}=4 \sin \gamma_{i}$;

- $\theta_{i}=\alpha_{i}+\rho_{i}\left|\gamma_{i}\right|$

- $\vec{\xi}_{i}=\left(\cos \theta_{i}, \sin \theta_{i}\right)$. 


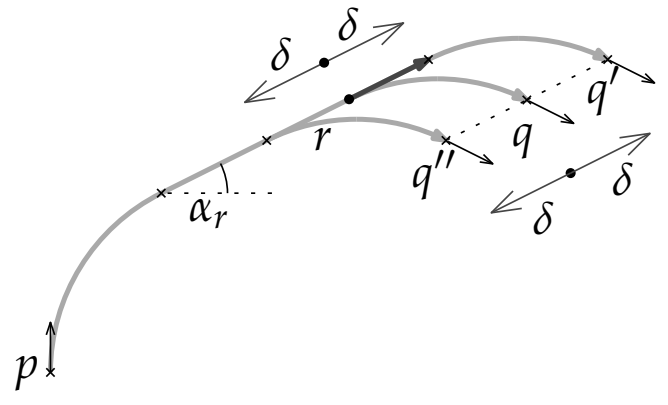

(a)

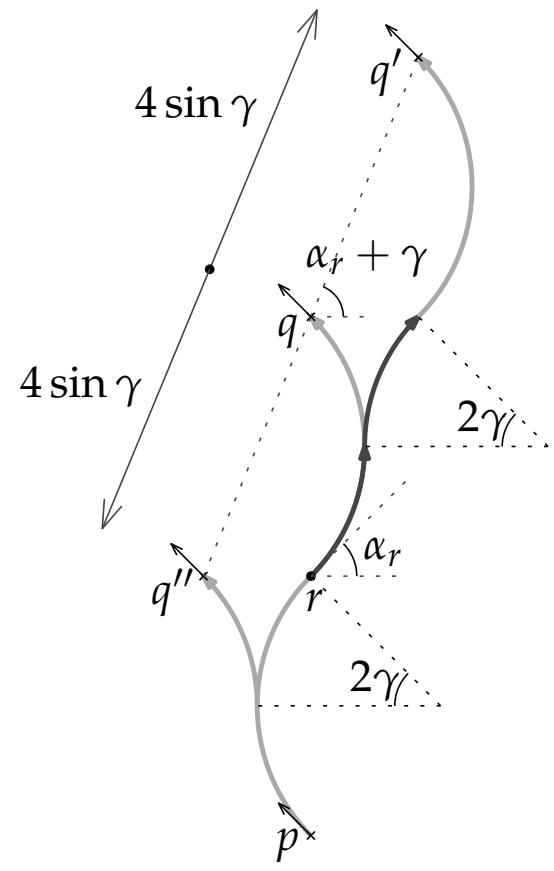

(b)

Figure 1: Examples of reversible deformations of CS-paths along $\mathcal{S}$ subpaths and at inflection points. In both examples, the original path from $p$ to $q$ is deformed reversibly into paths ending at $q^{\prime}$ (extension) and $q^{\prime \prime}$ (contraction). In (a), the $\mathcal{S}$ is simply extended or contracted by length $\delta$. In (b), however, the extension occurs by introducing two arcs of length $2 \gamma$ at $r$, while the contraction occurs by shortening the existing two arcs are shortened by $2 \gamma$ each, around $r$. 
Given a reversible direction $\left(\alpha_{i}, \rho_{i}\right)$, there exists $\varepsilon>0$ such that for $\gamma_{i} \in(-\varepsilon, \varepsilon)$ we can find a CS-path from the directed point $p$ to the directed point $q+\delta_{i}\left(\vec{\xi}_{i}, 0\right)$. Let $c(\alpha)$ denote the given directional cost function, and recall the definition of the cost of a path in Section 2.1. If $\rho=0$, the resulting change in cost is $\delta_{i} c\left(\alpha_{i}\right)$, otherwise it is $2 \int_{0}^{2 \gamma_{i}} c\left(\alpha_{i}+2 \rho_{i}|\phi|\right) \mathrm{d} \phi$. Note that $\gamma_{i}>0$ (extension) leads to an increase in cost, while $\gamma_{i}<0$ (contraction) leads to a decrease in cost.

The following Lemmas 2.4 and 2.7 were proven in [6].

Lemma 2.4 Let $A_{i}, A_{j}, A_{k}$ be three reversible directions of a CS-path from $p$ to q. Given any $\varepsilon>0$, there exist $\gamma_{i}, \gamma_{j}, \gamma_{k} \in(-\varepsilon, \varepsilon)$ such that the resulting path is distinct from the original but $q^{\prime}=q$, i.e.the following equation is satisfied with $\left(\gamma_{i}, \gamma_{j}, \gamma_{k}\right)$ non-zero:

$$
\delta_{i} \vec{\xi}_{i}+\delta_{j} \vec{\xi}_{j}+\delta_{k} \overrightarrow{\xi_{k}}=0
$$

Corollary 2.5 Let $A_{i}, A_{j}, A_{k}$ be three distinct reversible directions which lie in an open half-space of $\mathbb{R}^{2}$. Exactly one of these reversible directions, say $A_{j}$, is an interior reversible direction and there exist $\gamma_{i}, \gamma_{k}<0$ and $\gamma_{j}>0$ such that condition (2.1) is satisfied.

Definition 2.6 The velocity point of a reversible direction $A_{i}$ is the point in $\mathbb{R}^{2}$ which in polar coordinates is $\left(v\left(\alpha_{i}\right), \alpha_{i}\right)$.

Lemma 2.7 Let $A_{i}, A_{j}, A_{k}$ be distinct reversible directions which lie in an open half-space of $\mathbb{R}^{2}$ where $A_{j}$ is the interior reversible direction. Let $\rho_{i}, \rho_{j}, \rho_{k}=0$. Let the straight line passing through the velocity points of $A_{i}$ and $A_{k}$ be given in polar coordinates by the function $v_{i}^{k}(\alpha)$. There exist $\delta_{i}, \delta_{k}<0$ and $\delta_{j}>0$ satisfying (2.1) such that:

1. if $v\left(\alpha_{j}\right)>v_{i}^{k}\left(\alpha_{j}\right)$, then $\delta_{i} c\left(\alpha_{i}\right)+\delta_{j} c\left(\alpha_{j}\right)+\delta_{k} c\left(\alpha_{k}\right)<0$;

2. if $v\left(\alpha_{j}\right)<v_{i}^{k}\left(\alpha_{j}\right)$, then $\delta_{i} c\left(\alpha_{i}\right)+\delta_{j} c\left(\alpha_{j}\right)+\delta_{k} c\left(\alpha_{k}\right)>0$;

3. if $v\left(\alpha_{j}\right)=v_{i}^{k}\left(\alpha_{j}\right)$, then $\delta_{i} c\left(\alpha_{i}\right)+\delta_{j} c\left(\alpha_{j}\right)+\delta_{k} c\left(\alpha_{k}\right)=0$.

\section{Optimal Path Forms}

In this section, we study the form of optimal paths solving (1.1). The minimum length constraint means that it is possible that an optimal path may contain unit 
circles traversed along the path. Since these are excluded from the definition of a CS-path, we define a loop to be such an arc. Note that the location of a loop (or whether it is left or right-turning) does not affect the cost or feasibility of a CS-path with loops, and thus we do not need to specify where these loops occur along a particular curvature-constrained path. Given a CS-path $E$ and lower bound on length $L_{l b}$, it is clear that the correct number of additional loops to consider is the lowest number of loops required to make the CS-path with loops satisfy the minimum length constraint. This is the number of loops we imply when we refer to a CS-path with loops, which means that a CS-path with loops will not have any loops if the CS-path itself already satisfies the prescribed minimum length constraint. It is important to keep in mind that loops do not correspond to any of the $\mathcal{C}$ labels in the form of a CS-path. For example, a path consisting of a straight line segment, followed by a loop, and then another straight line segment is simply a CS-path of the form $\mathcal{S}$ with one additional loop (see for example Figure 7).

Our approach in establishing the optimal forms will be similar to [6]. We first show that there exists an optimal CS-path with loops, and then proceed to consider CS-path deformations in order to identify the optimal forms. The main result we prove in this section is that for any velocity function, there exists an optimal path of the form $\mathcal{C S C S C S C}$ (or degeneracy) with loops.

In [8], Pontryagin's Maximum Principle was used in order to show that there exists an optimal CS-path for the problem without the lower bound on length (see [1] for more background on optimal control). However, it is inconvenient to formulate (1.1) as a control problem since there is a minimum length constraint. We therefore consider an alternative approach which requires us to first focus on proving some basic properties of length preserving reversible CS-path deformations. While these results initially appear to only be applicable if we assume an optimal CS-path exists, we will actually be able to apply them to show that there always exists an optimal CS-path.

\subsection{Length Preserving Reversible Deformations}

Recall that the degree of a CS-path is the number of reversible directions it has. Also note that the addition of loops to a CS-path does not affect its degree. In [6], reversible deformations (see Section 2.2) were introduced in order to establish which forms are not optimal when there is no minimum length constraint. However, most of the lemmas proven in [6] are not directly applicable when the minimum length constraint is active in (1.1) since they involve deformations which potentially shorten the path. Hence, we need to consider a length preserving combination of reversible deformations. In Lemma 2.4, three reversible directions were shown to be sufficient 
to perform a combination of reversible deformations which resulted in a distinct path from the original while preserving both $p$ and $q$. We now show that four reversible directions are sufficient to perform such a combination of deformations which also preserves the length.

Recall that $\delta_{i}=4 \sin \gamma_{i}, \theta_{i}=\alpha_{i}+\rho_{i}\left|\gamma_{i}\right|$ and $\vec{\xi}_{i}=\left(\cos \theta_{i}, \sin \theta_{i}\right)$. Let $\overrightarrow{\xi_{i}^{*}}=$ $\left(\delta_{i} \cos \theta_{i}, \delta_{i} \sin \theta_{i}, 4 \mu_{i}\right)$, where $\mu_{i}=\sin \gamma_{i}$ if $\rho_{i}=0$ and $\mu_{i}=\gamma_{i}$ if $\rho_{i} \neq 0$. We introduce $\overrightarrow{\xi_{i}^{*}}$ for convenience, as we will need an additional equation for the length preserving condition.

Let $A_{i}, A_{j}, A_{k}, A_{l}$ be four reversible directions of a CS-path from $p$ to $q$. Consider a new CS-path formed by performing deformations of size $\gamma_{i}, \gamma_{j}, \gamma_{k}, \gamma_{l}$ along the respective reversible directions. The resulting path terminates at $q$ with the same length as the original path if $\gamma_{i}, \gamma_{j}, \gamma_{k}, \gamma_{l}$ satisfy the following condition:

$$
\overrightarrow{\xi_{i}^{*}}+\overrightarrow{\xi_{j}^{*}}+\overrightarrow{\xi_{k}^{*}}+\overrightarrow{\xi_{l}^{*}}=0
$$

Lemma 3.1 Let $A_{i}, A_{j}, A_{k}, A_{l}$ be four reversible directions of a CS-path from $p$ to q. Given any $\varepsilon>0$, there exist $\gamma_{i}, \gamma_{j}, \gamma_{k}, \gamma_{l} \in(-\varepsilon, \varepsilon)$ such that the resulting path is distinct from the original but $q^{\prime}=q$, and the total length of the resulting path is the same as the original.

Proof. If there exists a pair of repeated reversible directions, without loss of generality let them be $A_{i}$ and $A_{j}$. Since there exists $\varepsilon_{1}>0$ such that the deformations are valid for $\gamma_{i}, \gamma_{j} \in\left(-\varepsilon_{1}, \varepsilon_{1}\right)$, we can simply choose $\gamma_{i}=\left(\min \left\{\varepsilon_{1}, \varepsilon\right\}\right) / 2$, $\gamma_{j}=-\left(\min \left\{\varepsilon_{1}, \varepsilon\right\}\right) / 2, \gamma_{k}=0, \gamma_{l}=0$ to satisfy $(3.1)$.

Suppose that $\alpha_{i}, \alpha_{j}, \alpha_{k}$ are distinct. Note that this is stronger than stating that there are no repeated reversible directions, since a pair of repeated reversible directions requires that both $\alpha$ and $\rho$ are the same. Consider $F: \mathbb{R}^{3} \rightarrow \mathbb{R}^{3}$ defined as follows:

$$
F\left(\gamma_{i}, \gamma_{j}, \gamma_{k}\right)=\overrightarrow{\xi_{i}^{*}}+\overrightarrow{\xi_{j}^{*}}+\overrightarrow{\xi_{k}^{*}}
$$

The Jacobian of $F$ can be simplified as follows:

$$
J\left(\gamma_{i}, \gamma_{j}, \gamma_{k}\right)=4\left[\begin{array}{ccc}
\cos \left(\alpha_{i}+2 \rho_{i}\left|\gamma_{i}\right|\right) & \cos \left(\alpha_{j}+2 \rho_{j}\left|\gamma_{j}\right|\right) & \cos \left(\alpha_{k}+2 \rho_{k}\left|\gamma_{k}\right|\right) \\
\sin \left(\alpha_{i}+2 \rho_{i}\left|\gamma_{i}\right|\right) & \sin \left(\alpha_{j}+2 \rho_{j}\left|\gamma_{j}\right|\right) & \sin \left(\alpha_{k}+2 \rho_{k}\left|\gamma_{k}\right|\right) \\
\partial \mu_{i} / \partial \gamma_{i} & \partial \mu_{j} / \partial \gamma_{j} & \partial \mu_{k} / \partial \gamma_{k}
\end{array}\right]
$$

where $\partial \mu_{i} / \partial \gamma_{i}=\cos \gamma_{i}$ if $\rho_{i}=0$ and 1 if $\rho_{i} \neq 0$. 
The determinant of $J$ evaluated at the origin is given by

$$
\operatorname{det}(J)=64\left(\sin \left(\alpha_{k}-\alpha_{j}\right)-\sin \left(\alpha_{k}-\alpha_{i}\right)+\sin \left(\alpha_{j}-\alpha_{i}\right)\right),
$$

which can be simplified into the following form:

$$
\operatorname{det}(J)=256 \sin \left(\left(\alpha_{k}-\alpha_{j}\right) / 2\right) \sin \left(\left(\alpha_{j}-\alpha_{i}\right) / 2\right) \sin \left(\left(\alpha_{k}-\alpha_{i}\right) / 2\right),
$$

which is nonzero since $\alpha_{i}, \alpha_{j}, \alpha_{k}$ are all distinct. Since $J$ is continuous at the origin, by the Inverse Function Theorem, there exists $\varepsilon_{2}>0$ such that for all $\gamma_{l} \in\left(-\varepsilon_{2}, \varepsilon_{2}\right)$, there exist $\gamma_{i}, \gamma_{j}, \gamma_{k}$ such that (3.1) is satisfied.

This leaves only one case to consider, which is when $\alpha_{i}, \alpha_{j}, \alpha_{k}$ are not all distinct. However, since we can exclude the case where there are repeated reversible directions, it follows from the expression of $J$ given above that there exists $\varepsilon_{3}>0$ such that $\operatorname{det}(J) \neq 0$ for all $\left\|\left(\gamma_{i}, \gamma_{j}, \gamma_{k}\right)\right\| \in\left(0, \varepsilon_{3}\right)$, where $\|\cdot\|$ denotes the Euclidean norm. Since $F\left(\gamma_{i}, \gamma_{j}, \gamma_{k}\right)$ is an odd function (antipodal with respect to the origin), it then follows that the inverse exists, and hence there exists $\varepsilon_{2}>0$ such that for all $\gamma_{l} \in\left(-\varepsilon_{2}, \varepsilon_{2}\right)$, there exists $\gamma_{i}, \gamma_{j}, \gamma_{k}$ satisfying (3.1).

Now that we have proved that such a combination of four reversible deformations exists, we wish to apply these deformations to paths of degree $d \geq 4$ to show that they are either not optimal or can be reduced to a path with degree $d \leq 3$.

We first consider the case where all four reversible directions are distinct and correspond to $\mathcal{S}$ segments. The result we obtain will illustrate the technique which can then be generalised to the other cases. A sequence of $n$ distinct reversible directions $A_{1}, \cdots, A_{n}$ are in ascending order if there exists $\varepsilon>0$ such that $\forall$ $\eta_{1}, \cdots, \eta_{n} \in(0, \varepsilon), \alpha_{i}+\rho_{i} \eta_{i} \in\left(\alpha_{i-1}+\rho_{i-1} \eta_{i-1}, \alpha_{i+1}+\rho_{i+1} \eta_{i+1}\right)$ for $i=1, \cdots, n$ where $\alpha_{0}+\rho_{0} \eta_{0}=\alpha_{n}+\rho_{n} \eta_{n}$ and $\alpha_{n+1}+\rho_{n+1} \eta_{n+1}=\alpha_{1}+\rho_{1} \eta_{1}$. Note that if $\rho_{1}=\cdots=\rho_{n}=0$, this definition simply reduces to the directions $\alpha_{1}, \cdots, \alpha_{n}$ being in ascending order.

Lemma 3.2 Let $A_{i}, A_{j}, A_{k}, A_{l}$ be four distinct reversible directions of a CS-path such that $\rho_{i}, \rho_{j}, \rho_{k}, \rho_{l}=0$, and $A_{i}, A_{j}, A_{k}, A_{l}$ are in ascending order. If $\left(\delta_{i}, \delta_{j}, \delta_{k}, \delta_{l}\right) \neq$ 0 satisfies condition (3.1), then $\operatorname{sgn}\left(\delta_{i}\right)=-\operatorname{sgn}\left(\delta_{j}\right)=\operatorname{sgn}\left(\delta_{k}\right)=-\operatorname{sgn}\left(\delta_{l}\right)$.

Proof. Since $\rho_{i}, \rho_{j}, \rho_{k}, \rho_{l}=0$, we can simplify the length preserving condition (the equation corresponding to the third component in the system (3.1)) to the following: 


$$
\delta_{i}+\delta_{j}+\delta_{k}+\delta_{l}=0
$$

It is clear that it is impossible to satisfy (3.2) with the signs of $\delta_{i}, \delta_{j}, \delta_{k}, \delta_{l}$ being the same since $\left(\delta_{i}, \delta_{j}, \delta_{k}, \delta_{l}\right) \neq 0$.

Now suppose that three of $\delta_{i}, \delta_{j}, \delta_{k}, \delta_{l}$ have the same sign. Without loss of generality, we can let $\delta_{i}, \delta_{j}, \delta_{k} \geq 0$ and $\delta_{l} \leq 0$. From the first two equations of (3.1), we get that $\delta_{i} \vec{\xi}_{i}+\delta_{j} \vec{\xi}_{j}+\delta_{k} \vec{\xi}_{k}=\left|\delta_{l}\right| \vec{\xi}_{l}$. From (3.2), we get $\delta_{i}+\delta_{j}+\delta_{k}=\left|\delta_{l}\right|$. However, this is only possible if either $\vec{\xi}_{l}=\vec{\xi}_{i}, \vec{\xi}_{l}=\vec{\xi}_{j}$ or $\vec{\xi}_{l}=\vec{\xi}_{k}$, which contradicts the fact that the four reversible directions are distinct. Hence, it is not possible for three of the signs of $\delta_{i}, \delta_{j}, \delta_{k}, \delta_{l}$ to be the same.

Now suppose that $\delta_{i}, \delta_{j} \geq 0$ and $\delta_{k}, \delta_{l} \leq 0$. Let $L=\delta_{i}+\delta_{j}$, which by (3.1) implies that $L=\left|\delta_{k}\right|+\left|\delta_{l}\right|$. Let $X_{i j}$ denote the line segment in $\mathbb{R}^{2}$ from $L \vec{\xi}_{i}$ to $L \vec{\xi}_{j}$ and $X_{k l}$ denote the line segment in $\mathbb{R}^{2}$ from $L \vec{\xi}_{k}$ to $L \vec{\xi}_{l}$. From (3.1), we know that $\delta_{i} \vec{\xi}_{i}+\delta_{j} \vec{\xi}_{j}$ and $\left|\delta_{k}\right| \vec{\xi}_{k}+\left|\delta_{l}\right| \vec{\xi}_{l}$ are equal, and belong in $X_{i j}$ and $X_{k l}$ respectively. By definition, it can be seen that $X_{i j}$ and $X_{k l}$ are chords of a circle of radius $L$ centred at the origin as shown in Figure 2. Since $\alpha_{k} \notin\left(\alpha_{i}, \alpha_{j}\right)$ and $\alpha_{l} \notin\left(\alpha_{i}, \alpha_{j}\right)$, it follows by basic properties of circles that the chords $X_{i j}$ and $X_{k l}$ are disjoint. This implies that it is not possible for condition (3.1) to be satisfied. Hence, it is not possible to have the signs of both $\delta_{i}, \delta_{j}$ or both $\delta_{j}, \delta_{k}$ to the same.

This leaves the only possible scenarios which satisfy condition (3.1) to be $\delta_{i}, \delta_{k} \geq 0$ and $\delta_{j}, \delta_{l} \leq 0$, or $\delta_{i}, \delta_{k} \leq 0$ and $\delta_{j}, \delta_{l} \geq 0$, proving the result.

Recall that the velocity point of a reversible direction $A_{i}$ is the point in $\mathbb{R}^{2}$ which in polar coordinates is $\left(v\left(\alpha_{i}\right), \alpha_{i}\right)$. For clarity, refer to Figure 3(a) for a diagram illustrating $\alpha_{\text {diag }}$ defined in the following lemma.

Lemma 3.3 Let $A_{i}, A_{j}, A_{k}, A_{l}$ be four distinct reversible directions of a CS-path such that $\rho_{i}, \rho_{j}, \rho_{k}, \rho_{l}=0$, and $A_{i}, A_{j}, A_{k}, A_{l}$ are in ascending order. Let $\alpha_{\text {diag }}$ denote the direction of the vector $\left(\left|\delta_{i}\right| \vec{\xi}_{i}+\left|\delta_{k}\right| \vec{\xi}_{k}\right)$ where $\left(\delta_{i}, \delta_{j}, \delta_{k}, \delta_{l}\right) \neq 0$ satisfies condition (3.1). Let the straight line passing through the velocity points of $A_{i}$ and $A_{k}$ be given in polar coordinates by the function $v_{i}^{k}(\alpha)$, with $v_{j}^{l}(\alpha)$ defined analogously for $A_{j}$ and $A_{l}$. Extending along $A_{i}$ and $A_{k}$ while contracting along $A_{j}$ and $A_{l}$ in such a manner that condition (3.1) is satisfied will:

- reduce the total cost of the path if $v_{i}^{k}\left(\alpha_{\text {diag }}\right)>v_{j}^{l}\left(\alpha_{\text {diag }}\right)$;

- increase the total cost of the path if $v_{i}^{k}\left(\alpha_{\text {diag }}\right)<v_{j}^{l}\left(\alpha_{\text {diag }}\right)$;

- not change the total cost of the path if $v_{i}^{k}\left(\alpha_{\text {diag }}\right)=v_{j}^{l}\left(\alpha_{\text {diag }}\right)$. 


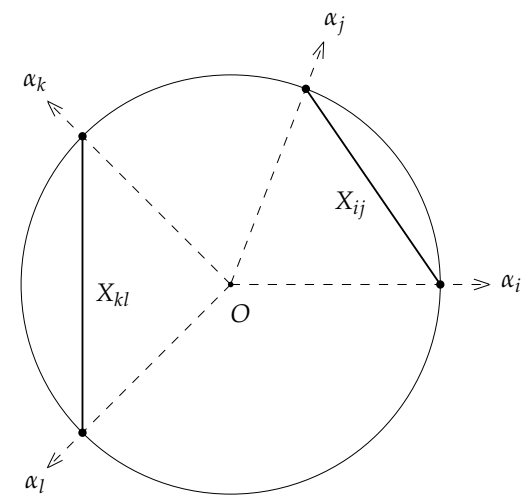

Figure 2: The chords $X_{i j}$ and $X_{k l}$ are disjoint since $\alpha_{i}, \alpha_{j}, \alpha_{k}, \alpha_{l}$ are arranged in ascending order. This basic property of circles is used in the proof of Lemma 3.2 to show that it is not possible for (3.1) to be satisfied with $\delta_{i}, \delta_{j} \geq 0$ and $\delta_{k}, \delta_{l} \leq 0$ where $\alpha_{i}, \alpha_{j}, \alpha_{k}, \alpha_{l}$ are arranged in ascending order.

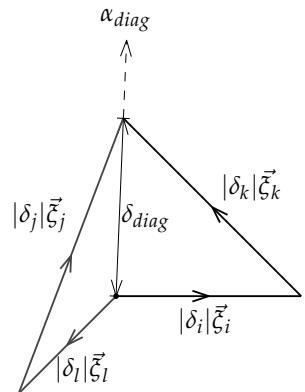

(a)

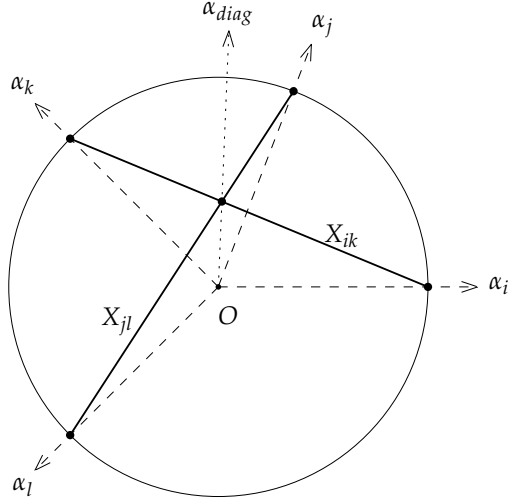

(b)

Figure 3: In (a), the vectors shown satisfy condition (3.1). A simple method for constructing a line in the direction $\alpha_{\text {diag }}$ is shown in (b) by locating the intersection of the two chords $X_{i k}$ and $X_{j l}$. 
Proof. Firstly, it follows from Lemma 3.2 that $\left|\delta_{i}\right| \vec{\xi}_{i}+\left|\delta_{k}\right| \vec{\xi}_{k}=\left|\delta_{j}\right| \vec{\xi}_{j}+\left|\delta_{l}\right| \vec{\xi}_{l}$ when condition (3.1) is satisfied. Let $\delta_{\text {diag }}=\left\|\left|\delta_{i}\right| \vec{\xi}_{i}+\left|\delta_{k}\right| \overrightarrow{\xi_{k}}\right\|$ where $\|\cdot\|$ denotes the Euclidean norm as shown in Figure 3(a).

By applying property (3) of Lemma 2.7, we know that the change in total cost of the path due to extending along $A_{i}$ and $A_{k}$ is equal to $\delta_{\text {diag }} / v_{i}^{k}\left(\alpha_{\text {diag }}\right)$. Similarly, the change in total cost of path due to contracting along $A_{j}$ and $A_{l}$ is equal to $-\delta_{\text {diag }} / v_{j}^{l}\left(\alpha_{\text {diag }}\right)$. The result then follows by summing these two changes in cost.

It is worth noting that $\alpha_{\text {diag }}$ can be obtained geometrically via the construction shown in Figure $3(\mathrm{~b}) . X_{i k}$ and $X_{j l}$ are line segments in $\mathbb{R}^{2}$ from $\vec{\xi}_{i}$ to $\overrightarrow{\xi_{k}}$ and from $\vec{\xi}_{j}$ to $\vec{\xi}_{l}$ respectively. It can be shown that $\alpha_{\text {diag }}$ is the direction from the origin $O$ to the intersection of the two chords $X_{i k}$ and $X_{j l}$.

The next step is to generalise Lemma 3.3 by removing the restriction of $\rho_{i}, \rho_{j}, \rho_{k}, \rho_{l}=0$.

Lemma 3.4 Let $A_{i}, A_{j}, A_{k}, A_{l}$ be four distinct reversible directions of a CS-path such that $A_{i}, A_{j}, A_{k}, A_{l}$ in ascending order. Let $\alpha_{\text {diag }}$ denote the direction of the vector $\left(\left|\delta_{i}\right| \vec{\xi}_{i}+\left|\delta_{k}\right| \vec{\xi}_{k}\right)$. Let the straight line passing through the velocity points of $A_{i}$ and $A_{k}$ be given in polar coordinates by the function $v_{i}^{k}(\alpha)$, with $v_{j}^{l}(\alpha)$ defined analogously for $A_{j}$ and $A_{l}$. There exists $\gamma_{i}, \gamma_{k}>0$ and $\gamma_{j}, \gamma_{l}<0$ satisfying condition (3.1) such that extending along $A_{i}$ and $A_{k}$ while contracting along $A_{j}$ and $A_{l}$ by the respective quantities will:

- reduce the total cost of the path if $v_{i}^{k}\left(\alpha_{\text {diag }}\right)>v_{j}^{l}\left(\alpha_{\text {diag }}\right)$;

- increase the total cost of the path if $v_{i}^{k}\left(\alpha_{\text {diag }}\right)<v_{j}^{l}\left(\alpha_{\text {diag }}\right)$.

Proof. The existence of $\gamma_{i}, \gamma_{k}<0$ and $\gamma_{j}, \gamma_{l}>0$ satisfying (3.1) is given by Lemma 3.2. The properties then follow by applying Lemma 2.4 with sufficiently small $\varepsilon$ so that the results hold in a similar manner to Lemma 3.3.

Note that there is a subtle difference between the conditions in Lemmas 3.3 and 3.4 above since $\alpha_{i}, \alpha_{j}, \alpha_{k}, \alpha_{l}$ are not necessarily distinct when $A_{i}, A_{j}, A_{k}, A_{l}$ are distinct. This, however, does not affect the validity of this result as if any of the directions $\alpha_{i}, \alpha_{j}, \alpha_{k}, \alpha_{l}$ are not distinct, then either $v_{i}^{k}\left(\alpha_{\text {diag }}\right)=v_{j}^{l}\left(\alpha_{\text {diag }}\right)$, or $\alpha_{\text {diag }}$ is not unique since the intersection is the entire chord, and any choice of $\alpha_{\text {diag }}$ will still satisfy $v_{i}^{k}\left(\alpha_{\text {diag }}\right)=v_{j}^{l}\left(\alpha_{\text {diag }}\right)$. 


\subsection{Existence of Optimal CS-path}

We have now developed the main tools required to prove that there exists an optimal CS-path solving (1.1). We first prove the following lemma which shows that, given a convex curvature-constrained path from $p$ to $q$, we can construct a CS-path of the same length between from $p$ to $q$. We will use this result to construct an arbitrarily close CS-path approximation to any given curvature-constrained path with the same length.

Lemma 3.5 Let $E$ be a convex left-turning curvature-constrained path from $p$ to $q$ such that the start and end directions $\alpha_{p}$ and $\alpha_{q}$ satisfy the condition that $\alpha_{q} \in$ $\left(\alpha_{p}, \alpha_{p}+\pi\right)$. If $E$ is not a $\mathcal{C S C}$ or $\mathcal{S C S}$ path, then there exists a $\mathcal{C S C S C}$ path $D$ from $p$ to $q$ such that $L(D)=L(E)$ and the direction of every point on $D$ lies in $\left[\alpha_{p}, \alpha_{q}\right]$.

Proof. Let $D_{1}$ be the $\mathcal{L} \mathcal{S} \mathcal{L}$ path from $p$ to $q$ and $D_{2}$ be the $\mathcal{S} \mathcal{L} \mathcal{S}$ path from $p$ to $q$. Note that $D_{1}$ and $D_{2}$ are left-turning curvature-constrained paths which enclose a region containing $E$. From basic properties of convex paths, it then follows that $L\left(D_{1}\right)<L(E)<L\left(D_{2}\right)$.

Let $O_{p}$ and $O_{q}$ denote the centres of the left-turning circles of unit radius corresponding to $p$ and $q$ respectively. Let $r$ denote the intersection of the ray from $O_{p}$ with direction $\alpha_{p}$ and the ray from $O_{q}$ with direction $\alpha_{q}+\pi$. Let $O_{p} r O_{q}$ denote the polygonal path from $O_{p}$ to $r$ to $O_{q}$. It is clear that $L\left(D_{1}\right)=L\left(O_{p} O_{q}\right)+\left(\alpha_{q}-\alpha_{p}\right)$ and $L\left(D_{2}\right)=L\left(O_{p} r O_{q}\right)+\left(\alpha_{q}-\alpha_{p}\right)$. Consider a family of polygonal paths made up of two straight lines, obtained by continuously deforming $O_{p} O_{q}$ into $O_{p} r O_{q}$ while fixing the start and end points at $O_{p}$ and $O_{q}$ respectively. It is clear that each of these polygonal paths $D_{p}$ corresponds to a $\mathcal{C S C S C}$ path $D$ from $p$ to $q$ with length $L\left(D_{p}\right)+\left(\alpha_{q}-\alpha_{p}\right)$ where the direction of every point on $D$ lies in $\left[\alpha_{p}, \alpha_{q}\right]$. It then follows by the fact that $L\left(D_{1}\right)<L(E)<L\left(D_{2}\right)$ that such a path $D$ exists with $L(D)=L(E)$.

For clarity of conveying the idea of the proof that there exists an optimal CS-path to (1.1), we will break this result up into two main steps. We first focus on showing that if there is an optimal convex curvature-constrained path from $p$ to $q$, then there exists an optimal CS-path of degree $d \leq 3$ from $p$ to $q$ with the same length as the convex path. We will then generalise this result to apply to any curvature-constrained path that is not necessarily a convex path.

Let the span of a path denote the smallest closed interval of directions which contains the directions of all points on the path. As stated in Section 2, we will assume that the size of the derivative of the directional cost function is bounded 
in order to simplify the following proof. In fact, we could easily even extend this to piecewise continuous function directional cost functions such that the derivative is bounded on each piece. This would simply involve breaking up the path into subpaths which only span a set of directions on which the directional cost function is continuous and applying the following proof. This makes it a reasonable condition to assume for any practical velocity function used in application.

Let $\mathbb{C}_{p q}$ denote the set of all CS-paths with loops from $p$ to $q$. Let $\mathbb{C}_{p q}^{3} \subset \mathbb{C}_{p q}$ denote the subset of paths with degree $d \leq 3$.

Lemma 3.6 Let $E$ be an optimal curvature-constrained path from $p$ to $q$. If $E$ is convex, then there exists an optimal path from $p$ to $q$ in $\mathbb{C}_{p q}^{3}$.

Proof. Suppose to the contrary that there is no optimal path in $\mathbb{C}_{p q}^{3}$. Since $E$ is a convex path, given any $\nu>0$, we can divide $E$ into $N_{\nu}$ subpaths $E_{i}$ for $i=0, \cdots, N_{\nu}$, such that each subpath $E_{i}$ is a convex path with the size of its span no greater than $\nu$. It is also clear that there exists $\hat{\nu}>0$ such that $N_{\hat{\nu}} \geq 4$ and $\hat{\nu}<\pi$.

Let $M$ be an upper bound on the derivative of the cost function $c(\alpha)$. By symmetry, we can assume that the subpath $E_{i}$ is left-turning. If $E_{i}$ is a $\mathcal{C S C}$ or $\mathcal{S C S}$ path then let $\hat{E}_{i}=E_{i}$. Otherwise, as long as $\nu<\pi$, we can apply Lemma 3.5 to show that there exists a $\mathcal{C S C S C}$ path $\hat{E}_{i}$ with the same length and span as $E_{i}$. This implies that $\left|T\left(\hat{E}_{i}\right)-T\left(E_{i}\right)\right| \leq \nu M L\left(E_{i}\right)$. We can apply this construction to all of the $N_{\nu}$ subpaths and construct a CS-path $E_{\nu}$ and obtain $\left|T(E)-T\left(E_{\nu}\right)\right| \leq$ $\sum_{i=1}^{N_{\nu}} \nu M L\left(E_{i}\right)=\nu M L(E)$.

Since there is no optimal path in $\mathbb{C}_{p q}^{3}$, given any $D \in \mathbb{C}_{p q}^{3}$, we know that $T(D)-T(E)>0$. We choose $\nu=\min \{\hat{\nu},(T(D)-T(E)) /(2 M L(E))\}$ so that $E_{\nu}$ is a CS-path with at least four distinct reversible directions $A_{i}, A_{j}, A_{k}, A_{l}$ where $\rho_{i}=\rho_{j}=\rho_{k}=\rho_{l}=0$ and $T\left(E_{\nu}\right)<T(D)$. Without loss of generality, we can assume $A_{i}, A_{j}, A_{k}, A_{l}$ are arranged in ascending order. By applying Lemma 3.3 to $T\left(E_{\nu}\right)$, we know that regardless of whether $v_{i}^{k}\left(\alpha_{\text {diag }}\right) \neq v_{j}^{l}\left(\alpha_{\text {diag }}\right)$ or $v_{i}^{k}\left(\alpha_{\text {diag }}\right)=v_{j}^{l}\left(\alpha_{\text {diag }}\right)$, we can construct a new CS-path of the same length and of no greater cost since the deformations considered are reversible. Since $E_{\nu}$ is itself a convex path by construction, performing this deformation until one of the straight line segments degenerates results in a new CS-path with 1 degree less. This procedure can be repeated, preserving the total length while never increasing the cost, until there are only 3 reversible directions remaining. Let $\hat{E}$ be this path, which by definition belongs in $\mathbb{C}_{p q}^{3}$. Hence, we have shown that for any path $E_{\nu} \in \mathbb{C}_{p q} \backslash \mathbb{C}_{p q}^{3}$, there exists a path $\hat{E} \in \mathbb{C}_{p q}^{3}$ such that $T(\hat{E}) \leq T\left(E_{\nu}\right)$. This contradicts our earlier statement that $T\left(E_{\nu}\right)<T(D)$, given any $D \in \mathbb{C}_{p q}^{3}$.

Hence, there exists an optimal path from $p$ to $q$ in $\mathbb{C}_{p q}^{3}$. 
A feasible curvature-constrained path to the problem (1.1) could, in general, have an infinite number of inflection points, as its only requirements are that it satisfies the curvature and minimum length constraints. In this paper, we only consider curvature-constrained paths with a finite number of inflection points. However, in [10], it is shown that we can always perturb a path with infinite inflection points into an arbitrarily closed path with finite inflection points. The techniques from that paper could be applied to generalise the following lemma to the case where we consider curvature-constrained paths with an infinite number of inflection points.

Lemma 3.7 Given any pair of directed points $p, q$, there exists an optimal path from $p$ to $q$ which is a CS-path with loops.

Proof. Let $E$ be an optimal curvature-constrained path from $p$ to $q$, possibly with an infinite number of inflection points. We can assume that $E$ is not a CS-path since the result follows trivially otherwise. Since $E$ is an optimal path from $p$ to $q$ which satisfies the minimum length constraint, we know that any subpath $E_{i}$ from $p_{i}$ to $q_{i}$ must also be an optimal path from $p_{i}$ to $q_{i}$ subject to a minimum length constraint equal to $L\left(E_{i}\right)$. As explained previously, we can assume that $E$ has a finite number, say $n$, of inflection points [10]. We can therefore choose to break $E$ into a finite number of subpaths $E_{i}$, separated by these inflection points, for $i=0 \cdots n$.

We can now apply Lemma 3.6 to each of the subpaths $E_{i}$, each of which is convex as it contains no inflection points, to deduce that there exists a path $D_{i} \in \mathbb{C}_{p q}^{3}$ which is also an optimal path from $p_{i}$ to $q_{i}$, with $L\left(D_{i}\right)$. Performing this on all the subpaths $E_{i}$ then results in a CS-path $D$ where $T(D) \leq T(E)$ and $L(D)=L(E)$. Hence, there exists an optimal CS-path with loops from $p$ to $q$.

\subsection{Sufficient Path Set}

Having proved that there exists an optimal CS-path, we now identify a sufficient set of CS-paths which contains an optimal path. We use the results developed in Section 3.1 to prove that there exists an optimal path with degree $d \leq 3$. Note that the addition of loops does not affect the degree of a CS-path. Hence, a path with $d \leq 3$ is equivalent to a path of the form $\mathcal{C S C S C S C}$ (or degeneracy) with loops. Recall that $\mathbb{C}_{p q}^{3}$ denotes the set of all CS-paths with loops between two directed points $p$ and $q$ with $d \leq 3$.

Theorem 3.8 Given any pair of directed points $p, q \in \mathbb{R}^{2} \times \mathbb{R} / 2 \pi \mathbb{Z}$ and $C^{0}$ cost function $c: \mathbb{R} / 2 \pi \mathbb{Z} \rightarrow \mathbb{R}_{+}$with bounded derivative, there exists an optimal path of the form $\mathcal{C S C S C S C}$ (or degeneracy) with loops. 
Proof. From Lemma 3.7, we know that there exists a CS-path $E$ which is optimal. Recall that a path in $\mathbb{C}_{p q}^{3}$ has degree $d \leq 3$ and hence, is a path of the form $\mathcal{C S C S C S C}$ (or degeneracy) with loops. Suppose to the contrary that $T(E)<T(D) \forall D \in \mathbb{C}_{p q}^{3}$. Since $E$ does not belong to $\mathbb{C}_{p q}^{3}$, it has degree $d \geq 4$. We now break the proof up into the following 3 subcases:

(1) $E$ has 4 distinct reversible directions $A_{i}, A_{j}, A_{k}, A_{l}$ arranged in ascending order such that $\alpha_{i}, \alpha_{j}, \alpha_{k}, \alpha_{l}$ are distinct;

(2) $E$ has 4 distinct reversible directions $A_{i}, A_{j}, A_{k}, A_{l}$ arranged in ascending order such that $\alpha_{i}, \alpha_{j}, \alpha_{k}, \alpha_{l}$ are not distinct;

(3) $E$ has a pair of repeated reversible directions $A_{i}, A_{j}$.

We explain case (1) in detail, after which cases (2) and (3) will hold via slightly modified arguments.

If (1) holds, and (adopting the notation used in Lemma 3.4 for velocity function $v=1 / c) v_{i}^{k}\left(\alpha_{\text {diag }}\right) \neq v_{j}^{l}\left(\alpha_{\text {diag }}\right)$, then it follows by Lemma 3.4 that $E$ is not optimal since there exists a path of lower cost than $E$ obtained through performing a combination of reversible deformations. Hence we can assume that the change in cost of any reversible deformations satisfying condition (3.1) is zero. This necessarily implies that $v_{i}^{k}\left(\alpha_{\text {diag }}\right)=v_{j}^{l}\left(\alpha_{\text {diag }}\right)$.

For the reversible direction $A_{i}$, let $I_{i}=\left(\alpha_{i}-\beta_{i}, \alpha_{i}+\beta_{i}\right)$ where $\beta_{i}>0$ is arbitrarily chosen to be sufficiently small so that $\alpha_{j}, \alpha_{k}, \alpha_{l} \notin I_{i}$. Let $h_{i}(\alpha)$ be defined as follows:

$$
h_{i}(\alpha)=\left\{\begin{array}{cl}
\beta_{i}-\left|\alpha-\alpha_{i}\right| & \text { if } \alpha \in I_{i} ; \\
0 & \text { otherwise. }
\end{array}\right.
$$

Consider a new directional cost function $\hat{c}(\alpha)=c(\alpha)+\varepsilon h_{i}(\alpha)$ with corresponding velocity function $\hat{v}=1 / \hat{c}$. By construction, the velocity points of $A_{j}, A_{k}, A_{l}$ are the same for both $v$ and $\hat{v}$ while the velocity point of $A_{i}$ is different. This implies that $\hat{v}_{i}^{k}\left(\alpha_{\text {diag }}\right) \neq \hat{v}_{j}^{l}\left(\alpha_{\text {diag }}\right)$, using analogous notation for velocity points corresponding to $\hat{v}$. Hence, $E$ is not an optimal path for $\hat{c}$, for any $\varepsilon>0$. Let $T(E)$ and $\hat{T}(E)$ denote the costs of path $E$ with respect to the directional cost functions $c$ and $\hat{c}$ respectively. Let $H(E)=\int_{E} h_{i}(\alpha) \mathrm{d} s$. Then: 


$$
\begin{aligned}
\hat{T}(E) & =T(E)+\varepsilon H(E) \\
& <T(D), \forall D \in \mathbb{C}_{p q}^{3}, \text { by choosing } \varepsilon \in(0,(T(D)-T(E)) /(H(E))) \\
& <\hat{T}(D), \forall D \in \mathbb{C}_{p q}^{3} .
\end{aligned}
$$

This results in a contradiction since $E$ is not an optimal path for the directional cost function $\hat{c}$.

If case (2) holds, we are unable to apply the exact same argument as for case (1), since introducing $h_{i}(\alpha)$ will not result in $\hat{v}_{i}^{k}\left(\alpha_{\text {diag }}\right) \neq \hat{v}_{j}^{l}\left(\alpha_{\text {diag }}\right)$. Without loss of generality, let $\alpha_{i}=\alpha_{j}$ and $\rho_{i} \neq 0$. If $\rho_{i}=1$, let $I_{i}^{+}=\left(\alpha_{i}, \alpha_{i}+\beta_{i}\right)$ and define $h_{i}^{+}(\alpha)$ as follows:

$$
h_{i}^{+}(\alpha)=\left\{\begin{array}{cl}
\beta_{i} / 2-\left|\alpha-\left(\alpha_{i}+\beta_{i} / 2\right)\right| & \text { if } \alpha \in I_{i}^{+} \\
0 & \text { otherwise }
\end{array}\right.
$$

Using $h_{i}^{+}(\alpha)$ in place of $h(\alpha)$ still gives $\hat{v}_{i}^{k}\left(\alpha_{\text {diag }}\right)=\hat{v}_{j}^{l}\left(\alpha_{\text {diag }}\right)$; however, there now exist sufficiently small reversible deformations satisfying condition (3.1) such that the change in cost $\hat{T}$ using $\hat{c}$ is non-zero, since $h_{i}^{+}(\alpha)$ only affects the cost of the extension (or contraction) along $A_{i}$ and does not affect the cost of the other reversible deformations. The rest of the argument then follows as before, since $E$ is not optimal for $\hat{c}$. It is then clear that if $\rho_{i}=-1$ we can let $I_{i}^{-}=\left(\alpha_{i}-\beta_{i}, \alpha_{i}\right)$ and define $h_{i}^{-}$in a similar manner to achieve the same result.

If case (3) holds, we first suppose that $\rho_{i}, \rho_{j}=0$. We can then simply extend the path along $A_{i}$ and contract it along $A_{j}$ in equal amounts until one of the straight line segments degenerates, resulting in a new distinct reversible direction or reducing the degree of the path by 1, while keeping the cost and length of the path constant. This procedure can be repeated until there are no pairs of repeated reversible directions $A_{i}, A_{j}$ where $\rho_{i}, \rho_{j}=0$. On the other hand, if $\rho_{i}, \rho_{j} \neq 0$, we can extend along $A_{i}$ and contract along $A_{j}$ in equal amounts without degenerating any of the existing arcs, in order to form a new path which still has the same pair of repeated reversible directions $A_{i}, A_{j}$ but with two additional distinct reversible directions. This procedure can then be repeated to produce a new path with the same length and cost as $E$ but with 4 distinct reversible directions, from which we can apply the arguments for case (1) or (2).

Hence, there exists an optimal path in $\mathbb{C}_{p q}^{3}$. 
Lemmas 3.1 - 3.7 and Theorem 3.8 all involve length preserving deformations. This guarantees that the length constraint is never violated. However, if the length constraint is inactive, then we are able to state more about the path as we can perform arbitrarily small deformations which change the length. For brevity, we refer the reader to [6] and state the following corollary with a sketch proof.

Corollary 3.9 Let $E$ be an optimal path such that $L(E)>L_{l b}$. There exists an optimal path of the form $\mathcal{C S C S C}$ (or degeneracy) with loops.

Proof. (Sketch) Since $L(E)>L_{l b}$, we are able to perform arbitrarily small CS-path deformations locally, even if the deformation is length reducing. This is useful as the most of the lemmas in [6] show that paths not of the form $\mathcal{C S C S C}$ (or degeneracy) are not optimal by performing small perturbations to the original path. In the other lemmas, a deformation which does not reduce the length of the path (nor increase the cost) is performed until the degree of the path is reduced (for some of the cases, a loop is created in this process). For our problem, this can be applied repeatedly to reduce a CS-path with loops down until we obtain an optimal path of the form $\mathcal{C S C S C}$ (or degeneracy) with loops.

\subsection{Necessary Optimality Condition}

Let $A_{i}, A_{j}, A_{k}$ be three reversible directions corresponding to a deformation with associated quantities $\gamma_{i}, \gamma_{j}, \gamma_{k}$ respectively such that (2.1) is satisfied and the change in cost is zero. It was shown in [7] that this necessarily implies the velocity function $v(\alpha)$ is a straight line passing through the three velocity points for $\alpha \in \Gamma_{i} \cup \Gamma_{j} \cup \Gamma_{k}$ where $\Gamma_{i}$ (and $\Gamma_{j}, \Gamma_{k}$ similarly) is defined as follows:

$$
\Gamma_{i}=\left\{\begin{array}{cl}
\left(\alpha_{i}-2\left|\gamma_{i}\right|, \alpha_{i}\right) & \text { if } \rho_{i}=-1 \\
\left\{\alpha_{i}\right\} & \text { if } \rho_{i}=0 \\
\left(\alpha_{i}, \alpha_{i}+2\left|\gamma_{i}\right|\right) & \text { if } \rho_{i}=1
\end{array}\right.
$$

This has also been used to prove the necessary optimality conditions for $C S$ paths in [7] and the relevance of the study of double tangents to the velocity function in finding the directions of the straights. We will now prove that when we consider length preserving deformations, the velocity points lie on a tangential conic section. Instead of a double tangent, this could be an ellipse (see for example Figure 5(a)), hyperbola (see for example Figure 5(b)), parabola or a simpler conic section such as a circle or straight line). Lemma 3.10 will illustrate the difficulty involved in constructing an optimal path of this form. 


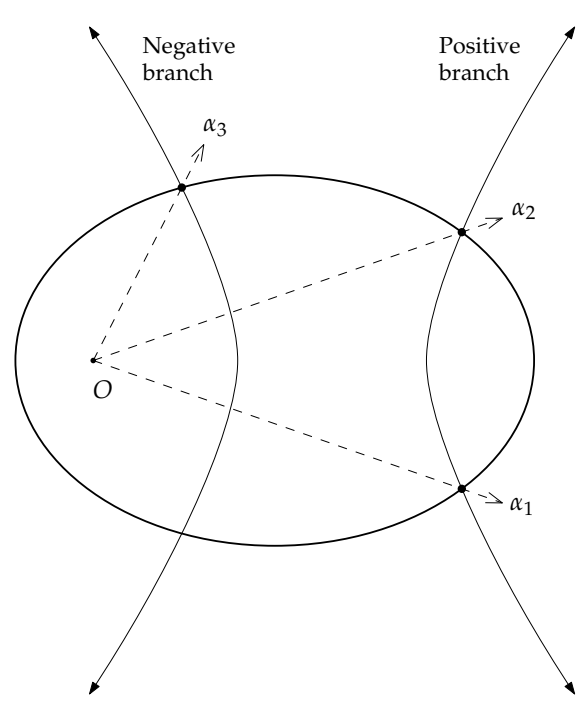

Figure 4: A unique conic section (ellipse) with focal point at the origin passing through the three velocity points in the directions $\alpha_{1}, \alpha_{2}, \alpha_{3}$. The hyperbola is the unique conic section passing through the velocity points in the directions $\alpha_{1}, \alpha_{2}$ and a negative point $\left(-v\left(\alpha_{3}\right), \alpha_{3}+\pi\right)$. This illustrates the importance of specifying that the velocity points are represented in polar coordinates, and not simply as points in $\mathbb{R}^{2}$.

Any conic section can be represented in polar coordinates in the form $r(\alpha)=$ $1 /(A+B \cos (\alpha)+C \sin (\alpha))$ with the origin at the focal point. By basic properties of conic sections, there exists a unique conic section passing through three given points in space, provided that the points are specified in polar coordinates as well, i.e. the points $(r, \alpha)$ and $(-r, \alpha+\pi)$ are distinct. This removes the possibility of having the negative branch of a hyperbola passing through any velocity points of a given velocity function $v$ since $v(\alpha)>0$ for all $\alpha \in \mathbb{R} / 2 \pi \mathbb{Z}$. An example illustrating the importance of this distinction is shown in Figure 4 where the ellipse is the unique conic section passing through the three velocity points. The hyperbola is invalid as it passes through the point $\left(-v\left(\alpha_{3}\right), \alpha_{3}+\pi\right)$ instead of $\left(v\left(\alpha_{3}\right), \alpha_{3}\right)$. This is why we require the values of the conic section to be positive when represented in the form $r(\alpha)=1 /(A+B \cos (\alpha)+C \sin (\alpha))$ in the following lemma.

Lemma 3.10 Let $A_{i}, A_{j}, A_{k}, A_{l}$ be distinct reversible deformations such that $\alpha_{i}, \alpha_{j}, \alpha_{k}, \alpha_{l}$ are distinct. Let $\Gamma_{i}$ be defined as in (3.3) above (and $\Gamma_{j}, \Gamma_{k}, \Gamma_{l}$ similarly). Given a velocity function $v=1 / c$, the change in cost of the reversible deformations $\gamma_{i}, \gamma_{j}, \gamma_{k}, \gamma_{l}$ satisfying (3.1) is zero if the points of $v(\alpha)$ for $\alpha \in \Gamma_{i} \cup \Gamma_{j} \cup \Gamma_{k} \cup \Gamma_{l}$ lie on the conic section with the focal point at the origin, represented in polar coordinates so that the values of the conic section are positive uniquely determined by three of the velocity points. 


\section{Proof.}

We will first show that if $\rho_{i}=\rho_{j}=\rho_{k}=\rho_{l}=0$, then the result holds. Suppose that $v\left(\alpha_{i}\right), v\left(\alpha_{j}\right), v\left(\alpha_{k}\right)$ are given but we are free to choose $v\left(\alpha_{l}\right)$. We wish to show that $v\left(\alpha_{l}\right)$ necessarily equals $\bar{v}\left(\alpha_{l}\right)$, where $\bar{v}(\alpha)$ is the conic section with a focal point at the origin that passes through the velocity points of $A_{i}, A_{j}, A_{k}$. By basic properties of conic sections, it follows that $\bar{v}(\alpha)$ is uniquely defined since $\alpha_{i}, \alpha_{j}, \alpha_{k}$ are distinct. For brevity, let $c_{i}$ denote $c\left(\alpha_{i}\right)$ and likewise for $j, k, l$. Since the change in cost is zero and (3.1) is satisfied, we obtain the following system of equations, where we arbitrarily set $\delta_{l}=1$ without loss of generality:

$$
\begin{aligned}
\delta_{i} \cos \alpha_{i}+\delta_{j} \cos \alpha_{j}+\delta_{k} \cos \alpha_{k}+\cos \alpha_{l} & =0 ; \\
\delta_{i} \sin \alpha_{i}+\delta_{j} \sin \alpha_{j}+\delta_{k} \sin \alpha_{k}+\sin \alpha_{l} & =0 ; \\
\delta_{i} c_{i}+\delta_{j} c_{j}+\delta_{k} c_{k}+c_{l} & =0 ; \\
\delta_{i}+\delta_{j}+\delta_{k}+1 & =0 .
\end{aligned}
$$

Note that the unknown parameters in the above system are $\delta_{i}, \delta_{j}, \delta_{k}, \alpha_{l}, c_{l}$ as everything else is assumed to be given. This can be simplified into the following system:

$$
\begin{aligned}
\cos \alpha_{l} & =\delta_{i}\left(\cos \alpha_{k}-\cos \alpha_{i}\right)+\delta_{j}\left(\cos \alpha_{k}-\cos \alpha_{j}\right)+\cos \alpha_{k} \\
\sin \alpha_{l} & =\delta_{i}\left(\sin \alpha_{k}-\sin \alpha_{i}\right)+\delta_{j}\left(\sin \alpha_{k}-\sin \alpha_{j}\right)+\sin \alpha_{k} \\
c_{l} & =\delta_{i}\left(c_{i}-c_{k}\right)+\delta_{j}\left(c_{j}-c_{k}\right)-c_{k} .
\end{aligned}
$$

Since a conic section has the general form of $r(\alpha)=1 /(A+B \cos (\alpha)+C \sin (\alpha))$, it is sufficient to show that there exist coefficients $A, B, C$ which are independent of $\delta_{i}, \delta_{j}, \delta_{k}, \alpha_{l}, c_{l}$, such that the following equation is satisfied:

$$
c_{l}=A+B \cos \alpha_{l}+C \cos \alpha_{l}
$$

Substituting (3.4), (3.5) and (3.6) into (3.7) gives:

$$
\left[\begin{array}{lll}
1 & \delta_{i} & \delta_{j}
\end{array}\right]\left[\begin{array}{c}
-c_{k} \\
c_{j}-c_{k} \\
c_{i}-c_{k}
\end{array}\right]=\left[\begin{array}{lll}
1 & \delta_{i} & \delta_{j}
\end{array}\right]\left[\begin{array}{c}
A+B \cos \alpha_{k}+C \sin \alpha_{k} \\
B\left(\cos \alpha_{k}-\cos \alpha_{i}\right)+C\left(\sin \alpha_{k}-\sin \alpha_{i}\right) \\
B\left(\cos \alpha_{k}-\cos \alpha_{j}\right)+C\left(\sin \alpha_{k}-\sin \alpha_{j}\right)
\end{array}\right]
$$


Since $\alpha_{i}, \alpha_{j}, \alpha_{k}$ are distinct, we can solve for $A, B, C$ via the following system of equations:

$$
\left[\begin{array}{ccc}
1 & \cos \alpha_{k} & \sin \alpha_{k} \\
0 & \cos \alpha_{k}-\cos \alpha_{i} & \sin \alpha_{k}-\sin \alpha_{i} \\
0 & \cos \alpha_{k}-\cos \alpha_{j} & \sin \alpha_{k}-\sin \alpha_{j}
\end{array}\right]\left[\begin{array}{c}
A \\
B \\
C
\end{array}\right]=\left[\begin{array}{c}
-c_{k} \\
c_{j}-c_{k} \\
c_{i}-c_{k}
\end{array}\right] .
$$

It is easily checked that the conic section $r(\alpha)=1 /(A+B \cos (\alpha)+C \sin (\alpha))$ does indeed pass through the velocity points of $A_{i}, A_{j}, A_{k}$ and hence $v\left(\alpha_{l}\right)=\bar{v}\left(\alpha_{l}\right)$.

The final step is to remove the restriction that $\rho_{i}, \rho_{j}, \rho_{k}, \rho_{l}=0$. It is sufficient to consider the cost of a convex subpath $E$ from $a \in \mathbb{R}^{2}$ to $b \in \mathbb{R}^{2}$, and show that if the velocity function is a conic section, then given any polygonal path $\hat{E}$ from $a$ to $b$ such that $L(\hat{E})=L(E)$, we obtain $T(\hat{E})=T(E)$. From the proofs of Lemmas 3.5 and 3.6, it follows that we can construct a polygonal path which is arbitrarily close to $E$. We can then apply the result we have just proved for $\rho_{i}=\rho_{j}=\rho_{k}=\rho_{l}=0$ repeatedly to any of these polygonal paths to show that they all have the same cost. Since we can create a sequence of polygonal paths of equal cost which in the limit converge to $E$, it follows that $T(E)=T(\hat{E})$.

We can now consider local perturbations to a $\mathcal{C S} \mathcal{S C S C}$ path with loops and non-degenerate straight line segments in a similar manner to [7] to obtain the following necessary optimality condition:

Corollary 3.11 If an optimal path contains an $\mathcal{S C S C S}$ path with the directions of the straights being $\alpha_{1}, \alpha_{2}$ and $\alpha_{3}$, then there exists $\varepsilon>0$ such that $v(\alpha) \leq \bar{v}(\alpha)$ for all $\alpha \in\left[\alpha_{1}-\varepsilon, \alpha_{1}+\varepsilon\right] \cup\left[\alpha_{2}-\varepsilon, \alpha_{2}+\varepsilon\right] \cup\left[\alpha_{3}-\varepsilon, \alpha_{3}+\varepsilon\right]$, where $\bar{v}(\alpha)$ is the unique conic section with a focal point at the origin that passes through the velocity points of $\alpha_{1}, \alpha_{2}, \alpha_{3}$.

Proof. Using techniques introduced in [7], we can consider a combination of deformations which perturbs $\alpha_{3}$ while preserving $\alpha_{1}, \alpha_{2}$ and the total length of the path. The result then follows from Lemma 3.10 and repeating the argument on all 3 directions $\alpha_{1}, \alpha_{2}, \alpha_{3}$.

As illustrated in Figures 5, unlike double tangents, it is much harder to find the number of triplet of points on the velocity function satisfying this condition, as conic sections could potentially be hyperbolas or ellipses. This makes the problem of constructing an optimal path of this form much more difficult. Furthermore, we have to also consider the cases where some of the straight line segments of the path are degenerate, resulting in separate necessary optimality conditions for these 
degenerate cases. This further complicates the procedure of constructing an optimal path of the form $\mathcal{C S C S C S C}$ (or degeneracy), even if we choose to restrict ourselves to considering piecewise constant velocity functions.

We can, however, consider the Lagrangian dual of the problem (1.1). This is an easier problem to solve as it can be reduced to a generalised version of the problem studied in [8], [6] and [7] which do not involve a length constraint. While it is unable to produce paths of the form $\mathcal{C S C S C S C}$, it is the logical first step of an algorithm attempting to solve (1.1) since it is much easier to solve, and in the event that it does not solve the problem, still provides a lower bound on the minimum cost of an optimal path. Details of this dual problem and its results can be found in [5]. Examples of the effectiveness and limitations of this approach are given in the next section.

\section{Examples}

As discussed in Section 3, it is difficult to construct all the feasibly optimal paths of the form $\mathcal{C S C S C S C}$ (or degeneracy) with loops. In this section, we present different examples to illustrate that in some scenarios, the Lagrangian dual approach can be very successful while in others, it fails to produce the optimal path. Since these examples involve comparing methods which solve different problems, we will refer to the problem we are interested in solving (1.1) as the primal problem, while the problem (1.1) without the minimum length constraint as the unconstrained problem and the Lagrangian dual problem (see [5] for more details) as the dual problem.

By weak duality [2], we know that the value of the objective function of the dual problem is a lower bound, $T_{\lambda}$, on the optimal value of the primal problem. This means that if we obtain a dual-optimal path $E^{*}$ such that $L\left(E^{*}\right)=L_{l b}$, then the primal problem is solved since $E^{*}$ is a feasible path to the primal problem with $T\left(E^{*}\right)=T_{\lambda^{*}}$. Example 1 below illustrates an example where this occurs.

Example 1: Consider the piecewise constant velocity function $v$ shown in Figure 6(a). Such velocity functions are important in practice as they correspond to piecewise constant cost functions. The optimal path $E$ from $p$ to $q$ for the unconstrained problem is shown in lighter grey in Figure 6(c). However, this path is not feasible for the given lower bound on length $L_{l b}$, i.e. $L(E)<L_{l b}$. The dual-optimal path $E^{*}$ is shown in darker grey where $L\left(E^{*}\right)=L_{l b}$. In [7], double tangents of the velocity function were shown to determine the optimal directions of the straight line segments. The procedure for solving the dual problem involves augmenting the given velocity function as shown in Figure 6(b) with the double tangent corresponding to the pair of directions of the straight line segments shown on the figure. By 


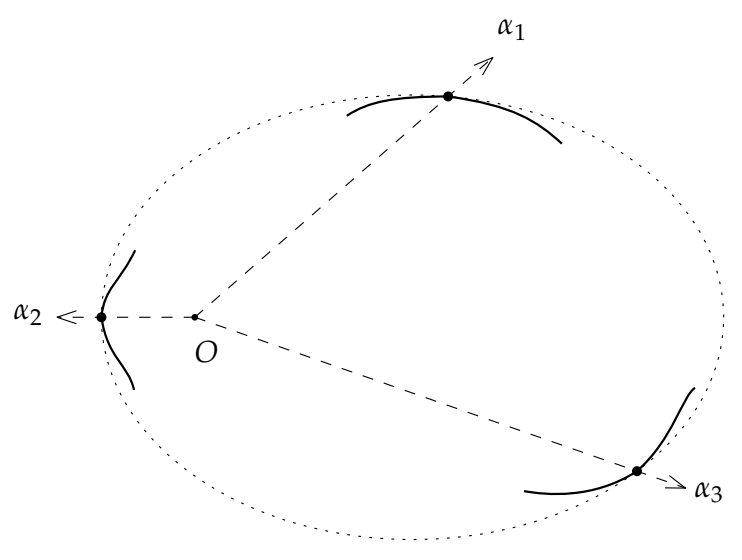

(a)

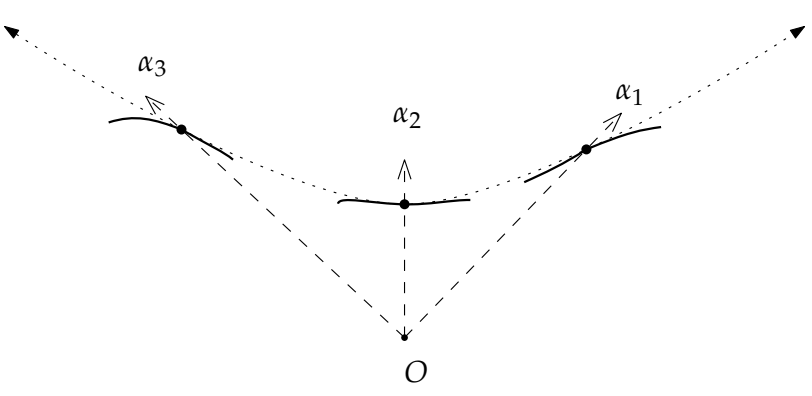

(b)

Figure 5: Illustration of Corollary 3.11 for an ellipse (in (a)) and a hyperbola (in (b)) with an exaggerated value of $\varepsilon$ for clarity.Each figure shows a plot of $v(\alpha)$ in thick solid line for neighbourhoods of size $\varepsilon$ about $\alpha_{1}, \alpha_{2}, \alpha_{3}$ with the conic section shown passing through the velocity points. 
weak duality, we conclude that the optimal path for the dual problem corresponds to an optimal path for the primal problem, in this example. It is worth noting that in this example, an optimal solution cannot be obtained by simply adding loops to optimal paths of the unconstrained problem.

The next example illustrates a case where the dual problem approach does not give us the primal optimal path since we only consider paths of the form $\mathcal{C S C S C}$ (or degeneracy) without loops.

Example 2: Consider a constant velocity function $v=1$ so that this is the special case of the primal problem where we are interested in finding the shortest path given a lower bound on length. Let $p=(0,0,0), q=(\varepsilon, 0,0)$ and $L_{l b}=2 \pi$. It is clear that for sufficiently small $\varepsilon>0$, the optimal path is simply an $\mathcal{S}$ path with a loop added as shown in Figure 7. In this case, the optimal path to the unconstrained problem with a loop added is the optimal path. The dual-optimal path is simply the straight line segment from $p$ to $q$ without a loop.

Lastly, it is clear that the dual approach will not be able to solve the primal problem when all primal optimal paths have the form $\mathcal{C S C S C S C}$ such as in Example 3 below.

Example 3: Consider the velocity function $v$ shown in Figure 8(a). The path $E$ from $p$ to $q$, shown in Figure $8(\mathrm{~b})$, has length $L(E)=L_{l b}$. Since $E$ can be rearranged to form a loop and the polygonal path abcd as shown, it is clear by the given $v$ that $E$ is an optimal path. It is also clear that there is no $\mathcal{C S C S C}$ (even with loops) path that is optimal. In this case, the dual approach will only be able to provide a lower bound on the optimal primal value since it only considers paths of the form $\mathcal{C S C S C}$ (or degeneracy). This is still useful as it provides a performance bound on any subsequent $\mathcal{C S C S C S C}$ (or degeneracy) path obtained via other methods.

Example 3 is also a simple illustration of Corollary 3.11 where the conic section is just a circle. For more complicated velocity functions, it would be difficult to construct tangential conic sections. Furthermore, it is not clear that there is a bound on the number of tangential conic sections even if we restrict ourselves to piecewise constant velocity functions.

It is also worth pointing out that the velocity function in Example 3 can be easily modified to permit optimal paths with more than $3 \mathcal{S}$ segments by increasing the number of velocity points touching the unit circle. However, the important result is that we can always reduce such a path down to an optimal $\mathcal{C S C S C S C}$ (or degeneracy) path with loops, by Theorem 3.8. 


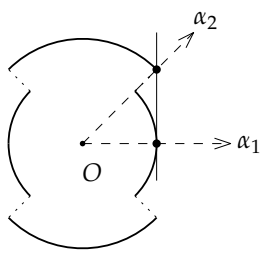

(a)
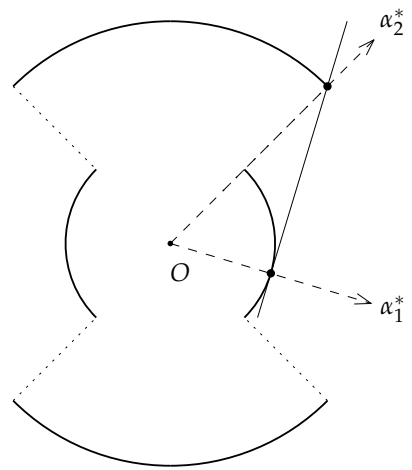

(b)

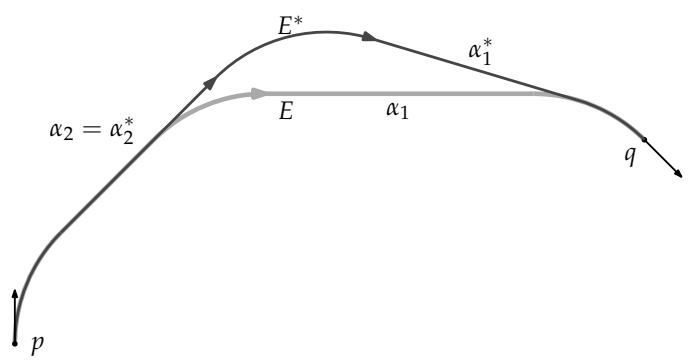

(c)

Figure 6: Illustration of Example 1 where the dual optimal path is also a primal optimal path, while the unconstrained optimal path is infeasible for the primal problem. Figure (a) shows a plot of $v(\alpha)=1 / c(\alpha)$ with a double tangent shown passing through the velocity points in directions $\alpha_{1}, \alpha_{2}$. Figure (b) shows a plot of $1 /\left(c(\alpha)-\lambda^{*}\right)$ with a double tangent shown passing through the velocity points in directions $\alpha_{1}^{*}, \alpha_{2}^{*}$. Figure (c) shows optimal $\mathcal{C S C S C}$ paths for the unconstrained problem and dual problem (shown in lighter grey and darker grey respectively) with the directions of the straight line segments annotated. 


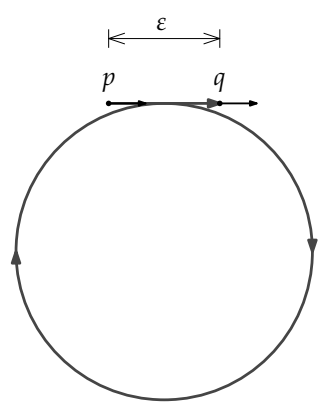

Figure 7: Illustration of Example 2 where the primal optimal path is a $\mathcal{S}$ segment with a loop. This form of path cannot be directly found by the dual approach.

\section{Conclusion}

In this paper, we have developed the theory necessary to produce paths which are directly applicable to the underground mine design problem. Within a particular geological domain where the support costs can be modelled as being primarily dependent on the direction in the horizontal projection, this model incorporates the essential vehicle navigability constraints of both maximum curvature and gradient. Due to the complicated nature of the gradient constraint, we are unable to present a simple algorithm which explicitly constructs an optimal path for a given set of initial conditions. However, we have proven that there exists an optimal path of the form $\mathcal{C S C S C S C}$ (or degeneracy) with loops. It is also possible to obtain a lower bound on the optimal value using Lagrangian duality, which requires solving a simpler problem. Further work would extend the existing theory to provide an explicit algorithm or heuristic for solving this problem in general.

\section{Acknowledgements}

This research is supported by a grant from the Australian Research Council.

\section{References}

[1] Ugo Boscain and Benedetto Piccoli, Optimal syntheses for control systems on 2-d manifolds, vol. 43, Springer, 2004.

[2] Stephen Boyd and Lieven Vandenberghe, Convex optimization, Cambridge University Press, 2004.

[3] M. Brazil, P. A. Grossman, D. H. Lee, J. H. Rubinstein, D. A. Thomas, and N. C. Wormald, Decline design in underground mines using constrained path 


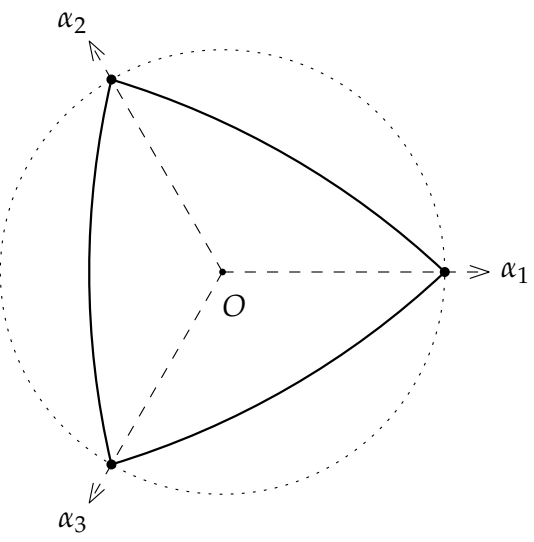

(a)

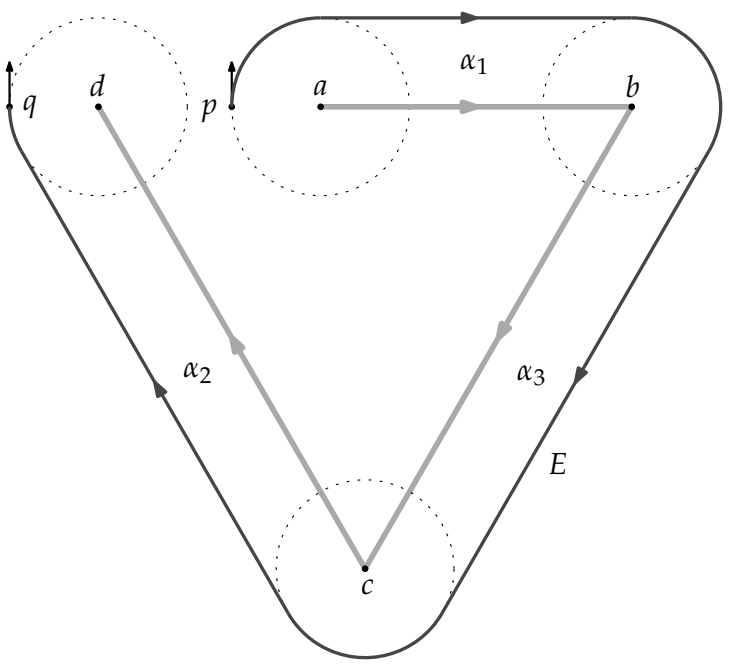

(b)

Figure 8: Illustration of Example 3 where the primal optimal path is of the form $\mathcal{C S C S C S C}$. Figure (a) shows the plot of $v(\alpha)=1 / c(\alpha)$ where $v\left(\alpha_{1}\right)=v\left(\alpha_{2}\right)=v\left(\alpha_{3}\right)$. Figure (b) shows the optimal $\mathcal{C S C S C S C}$ path for the primal problem, $E$, shown in darker grey with the polygonal path abcd formed by its 3 straight line segments shown in lighter grey. 
optimisation, Mining Technology: Transactions of the Institute of Mining and Metallurgy, Section A 117 (2008), no. 2, 93-99.

[4] M. Brazil and D. A. Thomas, Network optimization for the design of underground mines, Networks 49 (2007), no. 1, 40-50.

[5] A.J. Chang, Optimal curvature and gradient-constrained paths with anisotropic costs, Ph.D. thesis, Department of Mechanical Engineering, The University of Melbourne, Australia, Dec. 2011.

[6] A.J. Chang, M. Brazil, J.H. Rubinstein, and D.A. Thomas, Optimal curvatureconstrained paths for general directional-cost functions, Optimization and Engineering 14 (2013), 395-416.

[7] _ Constructing optimal curvature-constrained directional-cost paths, Engineering Optimization (submitted Jan. 2012).

[8] Alan Chang, Marcus Brazil, J. Rubinstein, and Doreen Thomas, Curvatureconstrained directional-cost paths in the plane, Journal of Global Optimization (2011), 1-19, 10.1007/s10898-011-9730-1.

[9] L. E. Dubins, On curves of minimal length with a constraint on average curvature, and with prescribed initial and terminal positions and tangents, American Journal of Mathematics 79 (1957), 497-516.

[10] J.A. Hoffman, M. Brazil, J.H. Rubinstein, and D.A. Thomas, A geometric approach to shortest curvature constrained paths in surfaces of constant curvature (working title), (In preparation).

[11] M. Fuchs K. Gehring, Quantification of rock mass influence on cuttability with roadheaders, 28th ITA (International Tunnelling Association) General Assembly and World Tunnel Congress. Sydney, 2002.

[12] D.H. Laubscher, A geomechanics classification system for the rating of rock mass in mine design, J. S. Atr. Inst. Min. Metal 90 (1990), no. 10, 257-273. 